Images du travail, travail des images

$8 \mid 2020$

Filmer, travailler, chercher

\title{
Filmer la performance ou l'irrévocabilité du travail
}

Filming the performance or the irrevocability of work

\section{Christian Lallier}

\section{CpenEdition}

\section{Journals}

Édition électronique

URL : http://journals.openedition.org/itti/379

DOI : $10.4000 /$ itti.379

\section{Éditeur}

Université de Poitiers

\section{Référence électronique}

Christian Lallier, «Filmer la performance ou l'irrévocabilité du travail », Images du travail, travail des images [En ligne], 8| 2020, mis en ligne le 01 février 2020, consulté le 14 avril 2021. URL : http:// journals.openedition.org/itti/379; DOI : https://doi.org/10.4000/itti.379

Ce document a été généré automatiquement le 14 avril 2021.

Images du travail, travail des images 


\title{
Filmer la performance ou l'irrévocabilité du travail
}

Filming the performance or the irrevocability of work

\author{
Christian Lallier
}

1 Filmer une situation de travail, c'est filmer ce qui se travaille dans une situation. Dans une perspective interactionniste, il s'agit de s'attacher aux relations sociales produisant une situation humaine : autrement dit, aux formes d'engagement par lesquelles les personnes se représentent dans le cadre d'une interaction selon la conduite de leur rôle. En portant son attention sur ce travail des relations sociales il devient possible de filmer ce qui se joue dans une situation de travail. C'est à partir de cette disposition que j'ai développé ma pratique de l'anthropologie par l'observation filmée : en m'attachant à la valeur performative d'une situation de travail, qu'il s'agisse, par exemple, de la pose d'un compteur électrique par un agent d'EDF ou de la fabrication de performances artistiques au Musée des Arts et Métiers, à Paris.

Il est convenu d'admettre qu'une interaction sociale implique au moins deux personnes. Pourtant, la simple relation aux objets du quotidien, par exemple, suppose un cadre d'engagement qui produit une situation sociale. En effet, chacun agit toujours selon des règles, des codes et des cadres d'action, et se réfère à des personnes, à des instances hiérarchiques et à toute présence qui se manifeste en son absence et avec laquelle il entretient un attachement. Filmer une telle situation implique de porter tout particulièrement son attention à l'état de réflexivité produit par l'engagement d'une personne dans son action. Je me souviens d'une circonstance de tournage m'ayant conduit à rendre compte de cette réalité sociale lors d'une situation de travail où la personne agissait seule. 


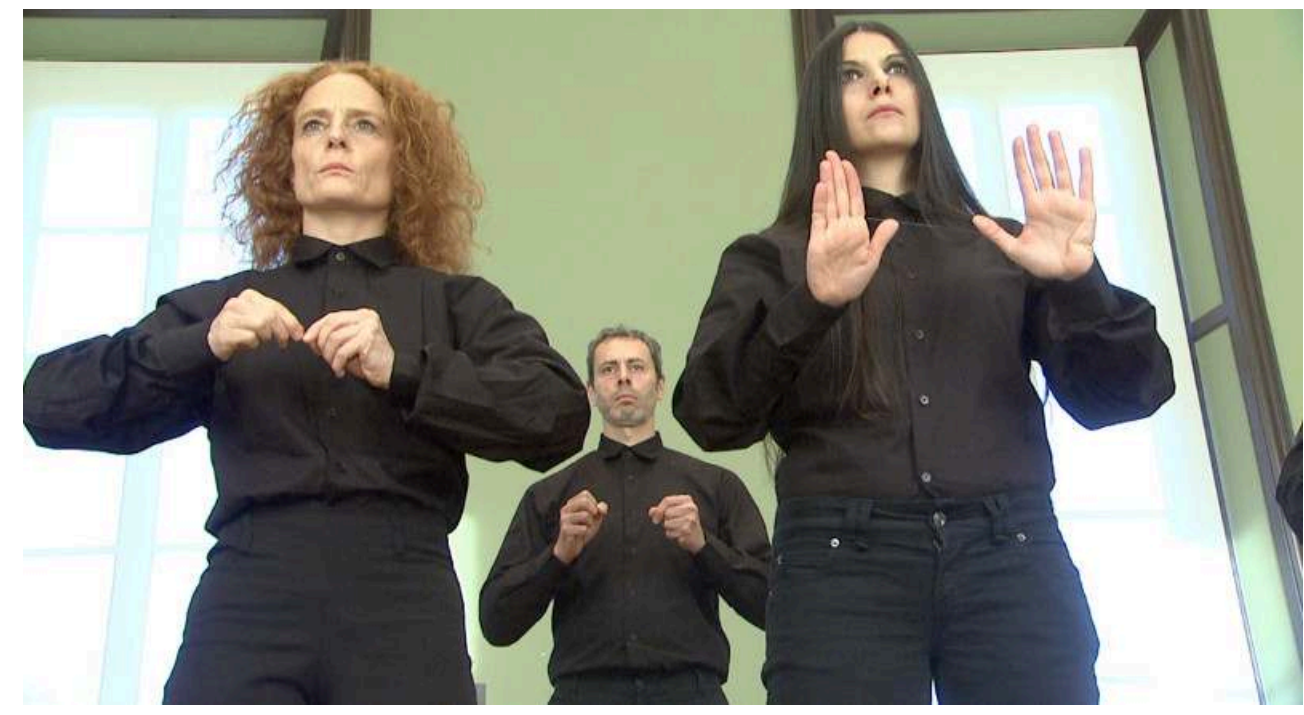

L'artiste italienne Romina de Novellis (à droite) avec deux autres artistes pendant sa performance ADN au Musée des Arts et Métiers à Paris.

Source : Performers\#10 - La fabrique de l'éphémère. Christian Lallier

\section{Le contre-monde d'une situation de travail}

3 L'une de mes toutes premières anthropologies filmées, menée à la fin des années 1990, avait pour objet le travail des agents EDF-GDF dans une commune de la région parisienne. Pendant une tournée, j'ai suivi l'un d'entre eux devant installer un compteur électrique chez un client: une situation d'apparence bien ordinaire, se déroulant selon une prescription très précise d'enchaînement des tâches. L'agent était donc seul. Pour autant, je lui ai posé un micro HF : je me doutais que le client viendrait, à un moment ou un autre, observer les travaux et qu'il échangerait avec l'agent installant le compteur dans le garage. Mais, je supposais surtout que cette prise de son au plus près de la personne engagée dans son action me permettrait de rendre compte de ce qui se jouait dans cette situation de travail. En effet, tout en filmant ses gestes et postures dans le suivi de l'exécution de sa tâche, je pouvais entendre ${ }^{1}$ non seulement sa respiration mais aussi lorsqu'il se parlait à lui-même : ce soliloque agissait comme un double de lui-même, contribuant au contrôle de l'action. À cet égard, rappelons que dans l'ancien français le "contre-rôle » désigne un "registre tenu en double $»^{2}$ pouvant servir à la vérification d'un acte. Selon cette même origine étymologique, le contrôle de soi s'interprète également comme une position réflexive permettant de vérifier la bonne conduite de son action (Lallier, $2008: 153-169)$. Le préfixe « contre » sous-entend une opposition entre deux forces contraires mais qui produit l'harmonie quand cette tension s'équilibre autour d'une forme ${ }^{3}$. 


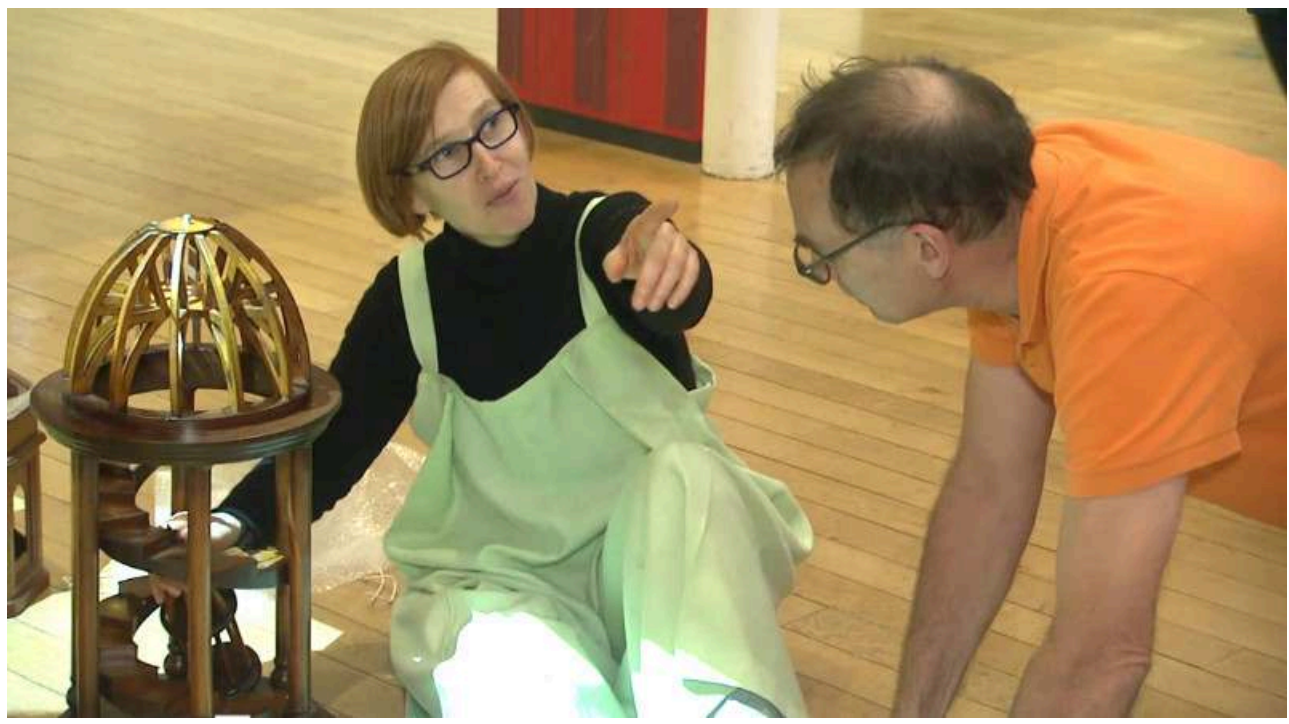

La danseuse et chorégraphe Cécile Proust pendant la préparation de sa performance avec son collaborateur, le vidéaste Jacques.

Source : Performers\#10 - La fabrique de l'éphémère. Christian Lallier

Cette notion de contrôle met en évidence un dédoublement de l'action par la dimension réflexive de toute situation de travail : autrement dit, comment nous contrôlons par nous-même nos propres actions. Cette dimension réflexive correspond au contremonde de la situation représentée, selon le terme proposé par Michel de Certeau : soit, un « revirement qui fait passer du recto au verso d'une société » (Certeau, 1973: 33), de l'environnement mondain de la situation (ce qu'il est convenu de voir) à l'écologie du cadre intime de l'action ${ }^{4}$. Le spectacle du cirque, par exemple, met précisément en représentation ce contre-monde par ses numéros qui donnent à observer le contrôle de soi des artistes. Le funambule, le trapéziste, le dompteur et, bien sûr, le clown sont autant de figures qui viennent rejouer chaque soir sous le chapiteau l'exercice de la condition humaine : être l'autre de soi-même pour agir en maîtrisant son destin. Cela suppose la mise en présentation de l'engagement (de soi) dans la représentation d'un script : autrement dit, en adoptant le déroulement d'un scénario pour le comédien, l'enchaînement d'un numéro pour le circassien ou la prescription d'une tâche pour l'opérateur d'une situation de travail, par exemple. Ainsi, chaque situation humaine, résulte-t-elle à la fois d'un cadre théâtral et d'un cadre social.

Reprenons l'exemple de la pose du compteur par l'agent EDF : cette situation constitue, d'une part, un fait social pouvant être représenté en tant qu'illustration typique d'une situation de travail d'un agent EDF et, d'autre part, une circonstance d'engagement pour cette personne dans la conduite de cette action. Nous pouvons donc remarquer que cette situation ne constitue pas un objet observable en soi, mais résulte d'un double phénomène qui se distingue par la possibilité ou non de le reproduire. En effet, ce que figure la situation peut faire l'objet d'une reconstitution, au sens que l'on peut reproduire la situation sous la forme d'un tableau en s'appliquant à re-fabriquer les différentes occurrences de la scène dans ses moindres détails, selon l'axe de vue par lequel nous l'avons initialement perçue et observée.

6 En revanche, la circonstance d'engagement ne peut être refaite à l'identique. Si j'avais demandé à l'agent d'EDF de reprendre tel geste ou telle posture, pour les besoins du 
film, j'aurais produit inévitablement une nouvelle circonstance d'engagement: soit, l'implication de la personne filmée pour les besoins du tournage et non pour la bonne conduite de son action, dans le cadre de son métier ${ }^{5}$. De même, si l'agent lui-même avait dû se reprendre suite à une erreur d'exécution, il aurait produit une nouvelle circonstance d'engagement, irréductible à la précédente, au principe qu'il devrait « reprendre » du temps pour agir, au risque -par exemple- de se mettre en retard. Cette double nature d'une situation se définit à la fois par le caractère réversible de son cadre de représentation -en tant qu'il est possible de reprendre l'action, comme tout comédien qui rejoue la même scène- et par l'irréversibilité de la circonstance d'engagement qui produit ce qui est vécu. Toute situation humaine relève ainsi de ce double état de représentation et d'engagement : une forme de bi-polarité qui pourrait s'apparenter au théâtre et son double décrit par Antonin Artaud, au sens où ce double correspondrait à l'état d'engagement produit par le cadre théâtral.

Image 3

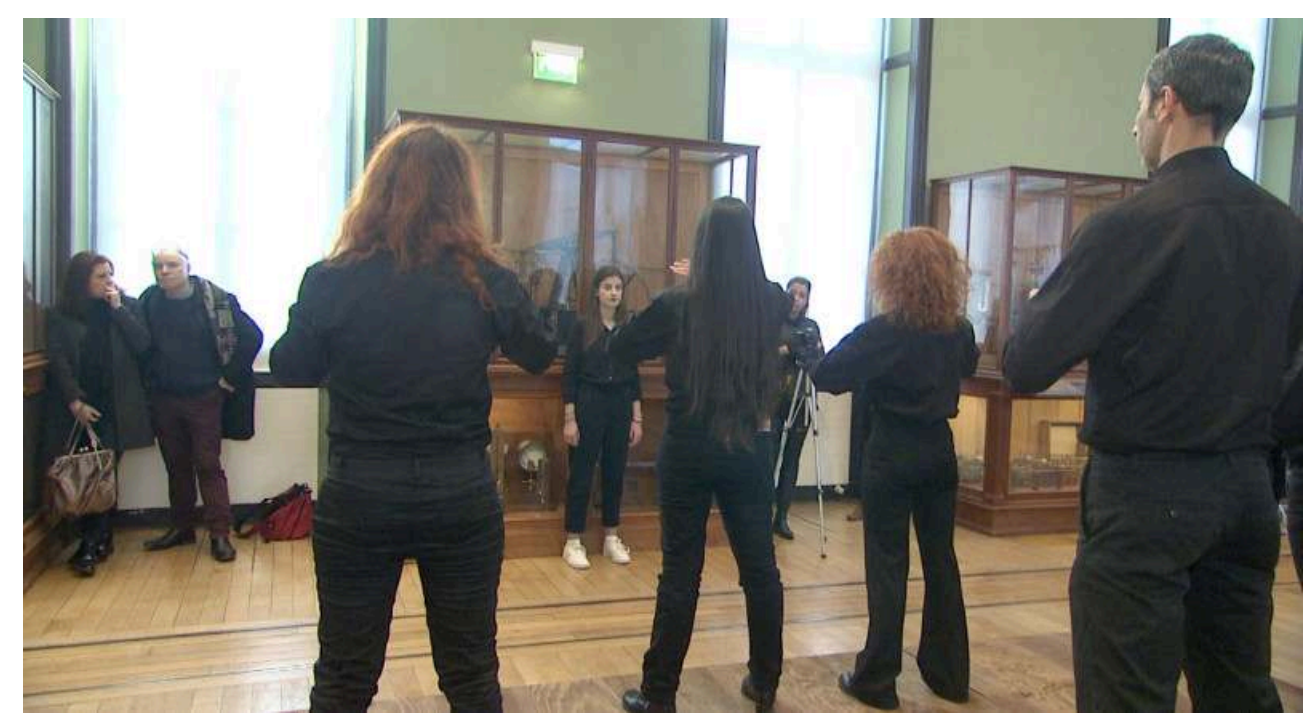

Le travail de la performance ADN, de Romina de Novellis devant le public.

La performance artistique met en regard le cadre social de l'engagement des artistes et le cadre théâtral de leur représentation.

Source : Performers\#10 - La fabrique de l'éphémère. Christian Lallier

Dans L'irréversible et la nostalgie, Vladimir Jankélévich analyse finement cette condition de l'existant (1974: 5) : "L'irréversible n'est pas un caractère du temps parmi d'autres caractères, il "est" la temporalitê même du temps; et le verbe "être" est pris ici au sens "ontologique" [...]. En d'autres termes il n'y a pas de temporalitê qui ne soit irréversible, et pas d'irréversibilité pure qui ne soit temporelle. La temporalité ne se conçoit qu'irréversible.» Or, si le réel se caractérise par l'irréversibilité de sa temporalité, alors cela signifie que la circonstance d'engagement - en tant qu'elle est irréversible - correspond au réel de la situation observée (Lallier, 2009 : 76-82).

Lors de l'observation filmée de la pose du compteur : ce qui m'était donné à voir, ce n'était plus la simple illustration de l'activité ordinaire d'un agent EDF, mais le temps irréversible d'une personne engagée dans l'irrévocabilité de son action. En cela, je ne m'attachais pas tant à la représentation du travail de l'agent -au produit du travailqu'à ce que pouvait représenter ce travail pour la personne elle-même -au travail produit par l'agent- ${ }^{6}$. 


\section{À la contiguïté de l'engagement et de la représentation}

En soutenant mon attention sur la circonstance d'engagement de la situation de travail, je m'attachais aux interactions: or, l'acte même de filmer m'aidait à observer les interactions entre l'agent EDF et les objets qu'il mobilisait pour agir ; entre ses mains se saisissant des outils et son regard contrôlant chaque geste ; entre les différentes zones composant l'espace de travail, depuis la camionnette servant d'atelier - garée à l'extérieur de la propriété du client - jusqu'au petit carré du mur du garage où était posé le compteur; etc. C'est l'observation filmée de toutes ces interactions qui me permettraient, au montage, de rendre compte de la situation en tant qu'elle était le produit de ces échanges. Pour le dire autrement, il ne s'agissait pas de filmer une situation pour montrer des interactions, mais de filmer des interactions pour montrer une situation. Cette conversion du regard suppose de s'attacher à la dimension performative de la situation de travail, autrement dit aux formes d'engagement d'une personne dans son action.

Image 4

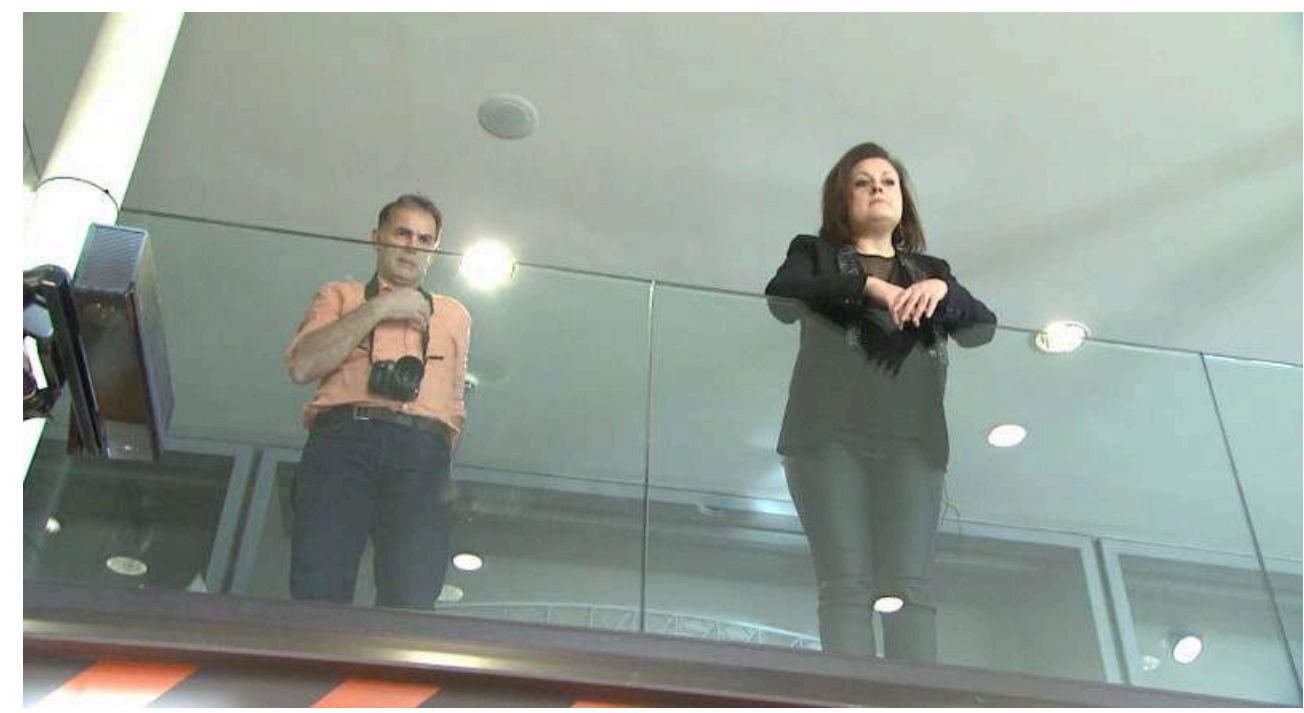

Nathalie Giuliana (à droite), en charge du projet Corps Dessinant au sein du Musée des Arts et métiers, avec le photographe-vidéaste et scénographe Jacques Hœpffner collaborant avec Cécile Proust pour sa performance.

Source : Performers\#10 - La fabrique de l'éphémère. Christian Lallier

Selon cette perspective, la séquence d'observation filmée de la pose du compteur EDF ne s'attachait pas à décrire la prescription de l'activité : elle visait à rendre compte des savoir-faire de l'agent qui se manifestent par des habilités mais aussi par des économies de l'effort qui impliquent des écarts à la règle; des formes d'ajustement aux consignes qui relèvent d'une compétence forgée par l'expérience; des modes d'action qui traduisent de l'ingéniosité et de la ruse dans la bonne conduite de ce qu'il est convenu d'exécuter.

11 Nous retrouvons, ici, la distinction entre « prescrit » et « réel » de l'ergonomie, visant à mettre en évidence « l'intelligence de la pratique » (la métis) de l'acteur devant réaliser une tâche (Dejours, 1995 : 49). Cette disposition conduit à s'intéresser aux différentes 
circonstances d'engagement d'interactions qui conduisent à l'accomplissement de l'action. Pour autant, la description de l'enchaînement des différentes occurrences composant le déroulement de la tâche ne suffit pas à rendre compte de la dimension performative de l'activité. Il convient également de porter l'attention sur le caractère irréversible de l'action-de chaque geste accompli, de chaque propos exprimé...-: autrement dit, de savoir rendre compte de la conduite d'une action, en tant qu'elle implique la responsabilité de celui qui agit vis-à-vis de tiers, présent(s) ou non lors de l'action. Si l'on rapporte ce cadre de description ethnographique à une situation d'échanges entre des personnes, on portera alors son attention au travail des relations sociales: en termes goffmaniens, on dira que l'on s'attache à la présentation de soi de chaque interactant qui s'implique dans une relation en face-à-face avec les autres personnes engagées dans la situation, en sorte qu'il puisse tenir son rôle selon l'ordre d'interaction qu'il convient de soutenir pour faire bonne figure et ne pas perdre la face (Goffman, 1996 : 29).

Bien qu'il soit seul dans son action, l'agent EDF installant le compteur doit garder la face vis-à-vis du client, de la hiérarchie et de ses collègues. Il doit s'engager à pouvoir se représenter selon un script instituant des codes, des normes et des règles, qui lui permettent d'être convenable dans la situation, au sens de savoir-faire bon voisinage avec les autres acteurs potentiels de la situation ${ }^{7}$. L'agent EDF se retrouvait ainsi en tension entre son engagement (personnel) et ce que représentait socialement sa situation de travail ${ }^{8}$ : c'est à la contiguïté de ces deux dimensions - engagement et représentation - que se produit la performance de l'acteur. Entre le théatre de la situation - au sens où le fait représenté pourra être "rejoué, " reconstitué, remis en scène - et la circonstance d'engagement - où se produit l'effort nécessaire à l'action -, s'exerce le travail des relations sociales : autrement dit, la dimension performative d'une situation humaine.

Image 5

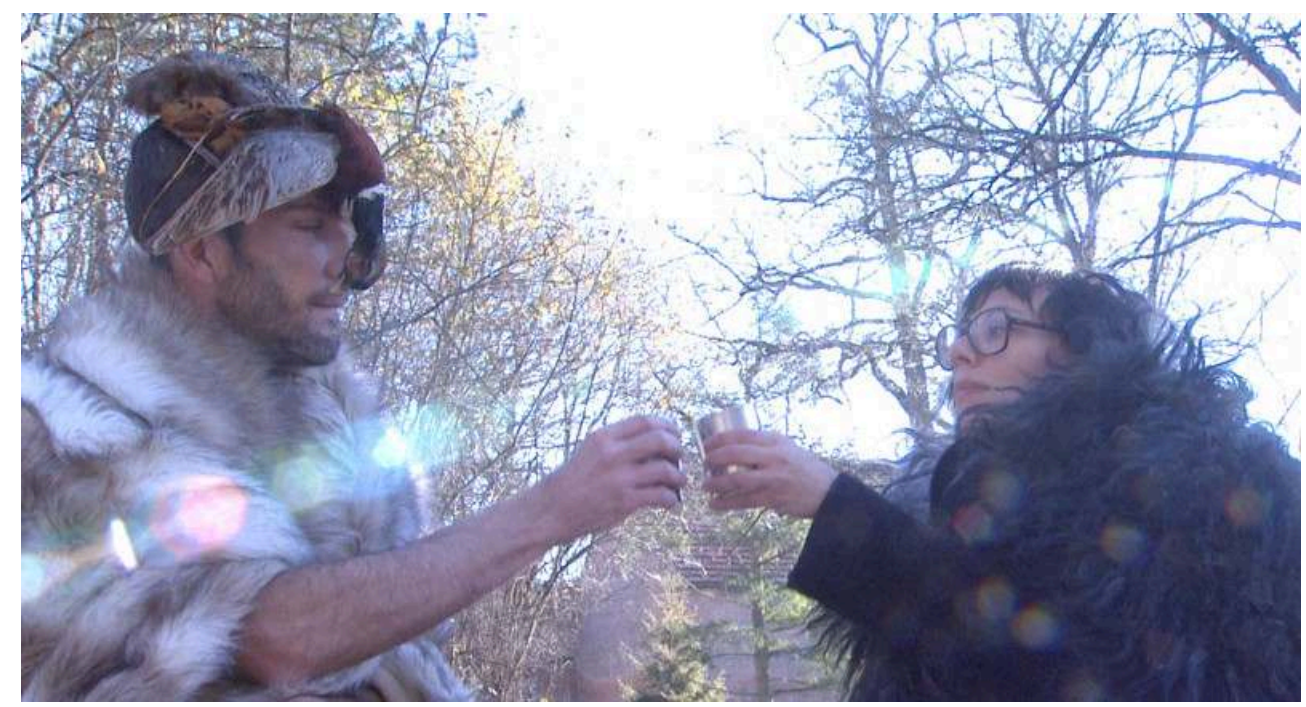

L'artiste-plasticien Julien Salaud et la danseuse Hélène Barrier se réchauffent avant de réaliser une chorégraphie sur un arbre : une séquence destinée à leur performance au Musée des Arts et Métiers. Source : Performers\#10 - La fabrique de l'éphémère. Christian Lallier

Dans le cas de la pose du compteur EDF, la dimension performative se donnait d'autant plus à voir que le temps de l'action était compté, qu'une économie du travail donnait toute 
la valeur à l'irréversibilité de chaque interaction: ce qui était fait n'avait pas à être refait, au risque sinon de ne pas respecter les délais. Précisons qu'à l'époque, lors de cette enquête de terrain, l'organisation du travail des agences EDF-GDF Services reposait sur une répartition des tâches entre les agents selon une mesure en poids horaire. En effet, chaque intervention était préparée par un agent qui devait notamment évaluer le temps nécessaire à l'exécution de la tâche : le responsable du planning de l'agence distribuait ensuite les tâches ainsi évaluées en fonction des tournées des agents. Or, pour cette tâche, le poids horaire avait été largement sous-évalué, de sorte que l'agent dû faire face à une pression de délais particulièrement stressante pour maintenir au mieux le planning prévu. En d'autres termes, la situation de travail ne se donnait plus à voir dans l'évidence d'une activité banale qui semble "aller de soi ", mais en tant qu'elle était une circonstance singulière, historiquement située, supposant une attention dans la conduite des actions afin de conduire à bien ce qui était convenu de faire, si possible, sans erreur ni échec. Dès lors, ce n'est pas tant la représentation de l'action qui constitue l'objet de l'observation filmée que la performance de l'acteur accomplissant cette action, au sens de performer (to perform), réaliser, ce qui doit être fait, en faisant preuve de compétence.

14 Cette précision apporte une première définition de la notion de performance en tant qu'elle désigne la mise en représentation de l'engagement d'un acteur dans la maitrise de son travail. Par exemple, si j'évoque la performance d'un acteur de théâtre je ne parle pas tant de la qualité de son jeu dans l'interprétation du rôle que de son implication personnelle dans la mise en représentation du personnage interprété. C'est l'engagement dans le travail qui devient, en soi, l'objet d'une représentation: la performance met en scène le réel qui est à l'œuvre dans l'objet représenté. En cela, on retrouve dans la performance artistique toutes les formes radicales de mise en visibilité de l'engagement irrévocable d'une personne dans son action : par exemple, la nudité et autres transgressions corporelles, l'épreuve du temps par la capacité à rester figé dans une posture (statuaire), l'expérience de l'espace (acrobatie), etc. De même, rendre compte de la dimension performative d'une situation de travail suppose de porter son attention sur le réel en tant que forme de représentation de l'action. Il ne s'agit pas d'observer une situation ordinaire dont l'engagement des acteurs produit un cadre théâtral - un fait de représentation qui potentiellement peut prendre la forme d'un tableau. Il ne s'agit pas non plus de regarder un cadre théâtral dont l'engagement des acteurs (des comédiens) produit un cadre social - l'interprétation d'une scène impliquant des relations de travail. Pour percevoir la dimension performative, il s'agit de considérer l'engagement des acteurs dans une situation théâtrale en tant que cette circonstance est bien réelle.

Imaginons que la situation de travail de la pose du compteur soit présentée sous la forme d'une installation dans un musée: l'agent EDF exécuterait sa tâche selon un temps imparti et qu'une horloge décompterait seconde par seconde. Cette mise en scène de l'engagement de l'agent EDF dans la présentation d'un script (la pose d'un compteur) produirait une performance. Le détournement de la situation de travail en une installation muséographique met en évidence le lien entre performance industrielle et performance artistique. Qu'il s'agisse d'une production industrielle ou d'une réalisation artistique, dans les deux cas, la dimension performative procède de la mise en représentation de l'irrévocabilité de l'acte exécuté : autrement dit, de ce qui qualifie le travail et le distingue de la nature des idées. Dans «Le chercheur et le 
quotidien », Alfred Schütz ${ }^{9}$ met en évidence cette distinction : « les actions simplement mentales sont révocables. Mais le travail ne l'est pas. Mon travail a transformé le monde extérieur. Au mieux, je puis restituer la situation initiale par des mouvements contraires, mais je ne puis défaire ce que j'ai fait. Voilà pourquoi je suis responsable d'un point de vue moral et légal - de mes actes et non de mes pensées » (Schütz, 1994 : 114-115). Cette définition phénoménologique du travail nous éclaire singulièrement sur ce que produit la performance : elle met en représentation l'engagement d'un individu dans une action qui produit un processus irréversible sur le monde. Dans le cas d'un artisan, sa performance consiste à maîtriser la transformation de la matière pour créer un objet. En effet, c'est bien le processus d'une opération technique qui conduit à modifier le monde : il y avait un bout de bois et dorénavant il y a une sculpture. Mais, le travail peut relever d'une opération qui vise à mettre en représentation l'engagement d'un individu dans l'exécution d'un acte de telle sorte que ce soit cette mise en représentation, en tant que telle, qui conduise à modifier le réel: dans ce cas, nous sommes en présence d'un rituel. Par exemple, lors de la fête des récoltes (Utchao) chez les Kalash dans les montagnes de l'Hindu-Kush au Pakistan, le chaman fait brûler du genévrier à l'attention du dieu Mahendéo ${ }^{10}$. Ce qui change le monde ce n'est pas tant de faire brûler du genévrier que la mise en représentation de l'engagement du chaman dans ce travail.

A la différence de l'artiste-performer, l'officiant -le prêtre- incarne par sa présence, ses gestes et sa parole, une autorité qui le transcende. En vertu de ce pouvoir qui lui est conféré, il pourra accomplir l'acte du baptême par le seul fait de dire, par exemple, «Je vous baptise » (Austin, $1970:$ 41-42). Cette efficacité symbolique procède de la mise en représentation de l'engagement du prêtre dans un acte de langage : dans une parole qui incarne un pouvoir. Quant au comédien jouant le rôle d'un prêtre qui baptise, sa performance n'aura aucun effet sur l'acte du baptême représenté (qui est une fiction) : en revanche, on pourra évoquer en quoi sa performance d'acteur a modifié le réel de son propre jeu... voire à modifier le monde (réel) du théâtre.

\section{Rendre compte d'une situation humaine}

17 À l'issue de cette analyse sur la notion de performance et sur ces différents usages dans le cadre social, théâtral ou religieux - on comprend qu'il n'y a pas fondamentalement de différence entre filmer l'exécution d'une tâche industrielle ou filmer une œuvre artistique.

18 Entre 2016 et 2018, j'ai réalisé une anthropologie filmée sur un programme de dix performances artistiques, un événement qui se déroula lors d'un week-end au musée des Arts et métiers, intitulé Corps dessinant ${ }^{11}$. Sous la forme d'une chronique ethnographique, cette anthropologie filmée retrace en sept épisodes le processus de création, le travail à l'œuvre et la présentation des performances. Cette série documentaire, intitulé Performers\#10 - La fabrique de l'éphémèr $e^{12}$, vise à rendre compte de la poïétique de ces performances, au sens où l'objet de recherche de cette anthropologie de la création «n'est ni l'œuvre à proprement parler, ni l'homme, ou le groupe producteur, mais les linéaments opératoires par lesquels l'œuvre vient (dans les meilleurs cas) à l'existence ", pour reprendre les termes de René Passeron (1996: 23). En s'attachant à ce qui se travaille dans la fabrication d'une création, cette anthropologie filmée s'apparente à une poïétique de la performance : il s'agissait d'explorer l'espace 
contigu qui se situe entre le monde créé et le contre-monde créateur et où se jouent les conditions d'émergence de l'œuvre. Porter l'attention sur les conditions de fabrication de l'œuvre, c'est aussi donner à voir les formes de négociation et les modes de justification par lesquels les interactants mettent en place un espace habitable pour agir ensemble.

Image 6

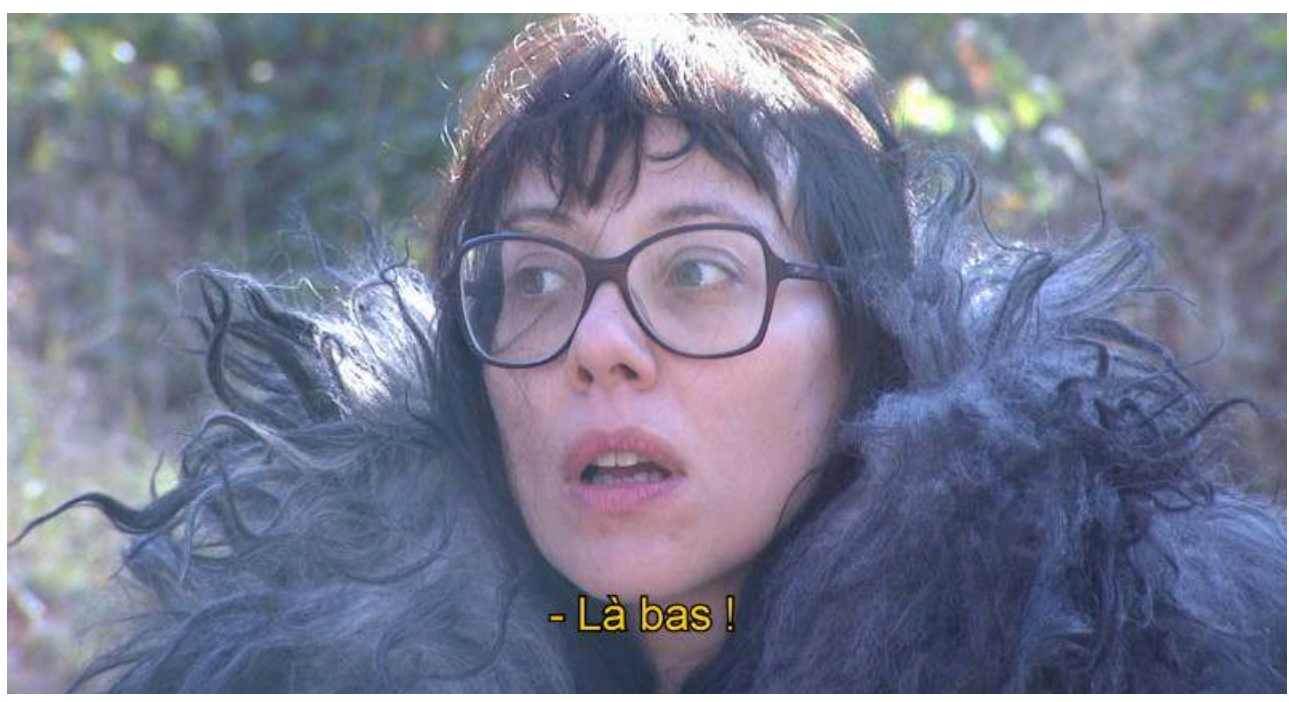

La danseuse Hélène Barrier.

Source : Performers\#10 - La fabrique de l'éphémère. Christian Lallier

19 Ces conditions de l'agir furent discutées lors d'une réunion de débriefing qui suivi le week-end de performances Corps dessinant. Performers\#10 se conclut par ce bilan : lors de cet échange, l'artiste-plasticien Richard Conte souligne en quoi cette expérience artistique, qu'il a co-organisée avec le Musée des Arts et métiers, relevait précisément d'une situation de travail : « Comme le dit Becker, dans Les mondes de l'art, la création ce n'est pas juste un artiste qui fait une œuvre, c'est en amont et en aval toute une série de corps de métiers, d'experts, de spécialistes, qui se mettent en œuvre pour qu'à un certain moment l'artiste puisse faire, par exemple là, sa performance. On voit rassemblé, au fond, ce que l'on ne voit jamais, tout l'invisible de la création. » Puis, à propos de la conduite de ce projet artistique, Richard Conte ajoute : "C'était un bel exemple de ce que nous appelons, nous, la "poḯtique", c'est-à-dire, l'étude de la création. Parce que l'étude de la création, elle n'échappe pas à toutes ses contraintes financières, organisationnelles, de sécurité, etc. Et la dimension de singularité, d'unicité de l'œuvre et ce à quoi nous avons assisté hier de très précieux, qui est l'acmé, c'est juste la partie visible de l'iceberg. » L'anthropologie filmée s'inscrit pleinement dans cette approche holistique qui consiste à interpréter un événement, aussi ordinaire soitil, dans la complexité des conditions de sa production.

Pour rendre compte du processus de création des performances de Corps dessinant, de leur poïétique, il convenait de s'attacher à la dimension performative des situations de travail qui produisaient ces objets artistiques: telle était mon intention pour la réalisation de cette anthropologie filmée. En d'autres termes, il s'agissait d'observer le travail des relations sociales entre les interactants, ce qui supposait de s'investir dans chacune de ces circonstances d'engagement. Il n'y avait pas, en soi, une situation de 
travail ou un type de situations de travail propre à la fabrication des performances. Cette approche ethnographique des interactions impliquait de s'adapter à la singularité de chacune des situations, aussi différentes les unes des autres que pouvaient l'être les performances qu'elles produisaient.

Il n'y avait donc pas une méthode spécifique pour filmer une catégorie de situation, comme s'il y avait une manière de filmer le travail, filmer la ville, filmer la mode ou les courses de chevaux. En revanche, quels que soient les contextes d'activités, il existe des méthodes d'enquête filmique différentes pour rendre compte des situations humaines. L'anthropologie filmée qui s'attache au travail des relations sociales (Lallier, 2009: 89-93), propose une méthode, une démarche qui vise à rendre compte d'une situation humaine par l'observation filmée de ce qui se joue entre les acteurs. Pour l'observateurfilmant, cela suppose d'adopter une posture de " petit » vis-à-vis des personnes filmées, autrement dit de n'apparaître ni comme une menace ni comme une ressource dans la situation sociale observée. Dès lors, il convient non seulement de ne pas intervenir dans le cours de l'action, mais de savoir aussi se mettre en mesure avec le rythme des échanges pour être en mesure d'en rendre compte. Il s'agit de prendre au sérieux non pas tant ce qui se dit mais ce qui se joue entre les acteurs, en sachant percevoir la valeur d'investissement des personnes engagées dans leurs actions. Selon cet état de perception, l'observateur-filmant se laisse embarquer par la circonstance d'engagement, tout en maîtrisant la conduite de l'observation filmée avec les contraintes et les impératifs techniques que cela suppose. On comprend alors que l'activité de tournage proprement dite ne soit plus un biais à l'observation mais au contraire contribue à l'intégration de l'observateur-filmant ${ }^{13}$ dans la situation sociale observée. Cette disposition particulière permet de filmer une situation humaine sans en modifier le cadre d'engagement ${ }^{14}$. Une telle condition de possibilité repose sur l'attention portée par l'observateur-filmant à l'investissement des personnes dans l'activité par laquelle elles sont représentées : cet intérêt pour l'engagement produit une plus-value sur la représentation des personnes en tant qu'elles s'investissent dans la conduite d'une action. Cette attention contribue ainsi à leur reconnaissance, voire à leur appartenance au groupe social observé. C'est par l'équilibre de ce rapport d'attention et de reconnaissance que les personnes se laissent filmer pour ce qu'elles représentent en tant qu'elles sont engagées dans la circonstance observée: c'est précisément en raison de cette disposition que les personnes ne chercheront pas à se mettre en scène pour la caméra puisque cela impliquerait immédiatement de leur part de se désengager de la circonstance par laquelle elles sont représentées et reconnues dans leur investissement ${ }^{15}$. 


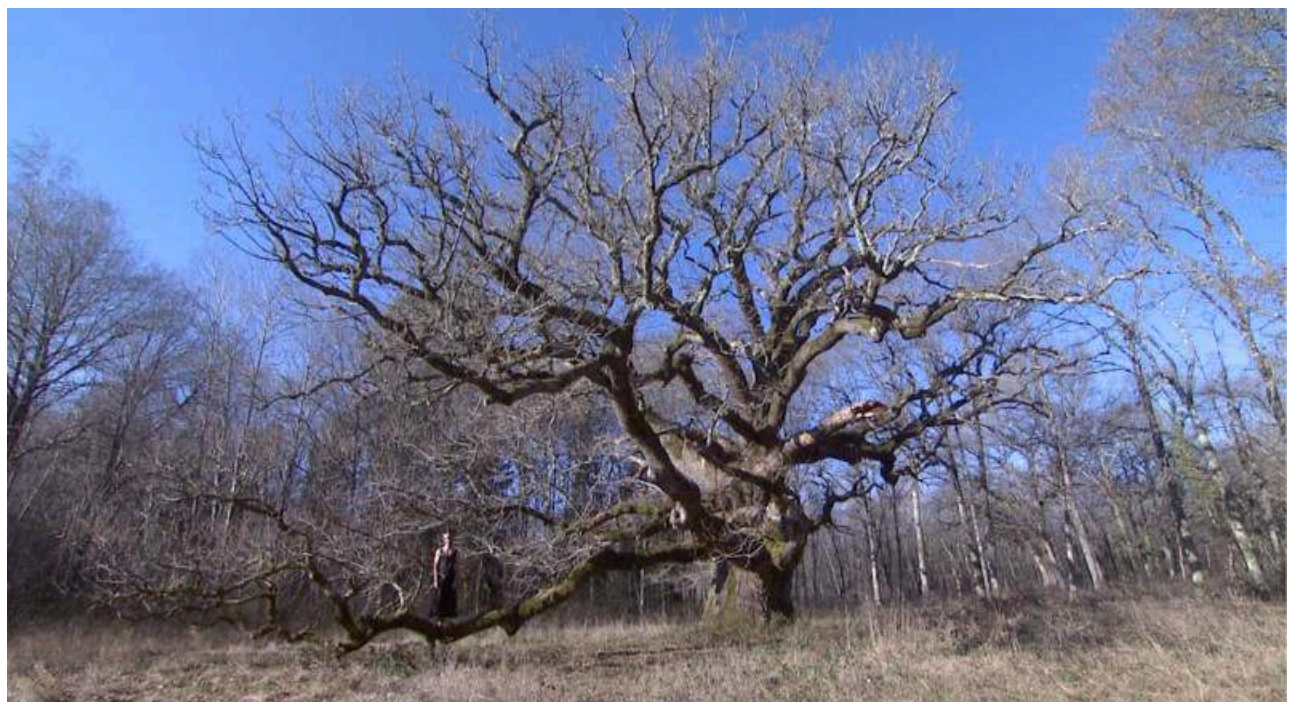

Un arbre dans la clairière d'une forêt en Sologne : lieu improbable d'une performance de Julien Salaud et d'Hélène Barrier. Julien Salaud sur l'arbre s'apprête à réaliser sa performance qui sera filmée par sa partenaire. La vidéo ainsi réalisée sera projetée lors de la performance en direct dans le Musée des Arts et Métiers à Paris.

Source : Performers\#10 - La fabrique de l'éphémère. Christian Lallier

En revanche, si une personne ne perçoit aucun intérêt à être engagée dans telle circonstance (qu'elle ne s'y sent pas à sa place, par exemple) ou qu'elle n'y est pas réellement engagée en raison de son statut - un cadre hiérarchique, par exemple, qui est juste de passage dans une réunion -, alors cette personne pourra refuser d'être filmée ou, si elle ne le peut pas, pourra fabriquer une mise en scène afin de donner un cadre de représentation satisfaisant à sa présence.

Cette fabrication peut aussi prendre la forme d'une plaisanterie à l'égard de l'observateur-filmant. Dans Les cadres de l'expérience, Erving Goffman définit la "fabrication" au cours d'une interaction sociale comme le jeu d'une feintise, tel que " "faire marcher" quelqu'un » ou «le piéger le temps d'une expression, d'une phrase, ou d'un tour de parole, puis à le mettre aussitôt au courant " (Goffman, 1991 : 98). Une autre forme de fabrication, propre à cette relation sociale singulière que représente le rapport filmant-filmés, ce sont les regards-caméras: ils surviennent lorsqu'une ou plusieurs personnes ne sont pas encore ou ne sont plus engagées dans la situation pour laquelle il est convenu (implicitement ou explicitement) qu'elles soient filmées. La présence des regards-caméras met en évidence le cadre d'engagement du rapport filmant-filmé qui ne peut s'interpréter comme une simple relation binaire reposant sur un dispositif technique ${ }^{16}$ : il convient d'y intégrer une relation à un tiers, terme par lequel filmant et filmé peuvent construire une relation sociale leur permettant d'agir ensemble, quand bien même il s'agirait d'une non-interaction où tout se passe comme si la personne ne savait pas qu'elle était filmée... : une disposition qui suppose que l'observateur-filmant puisse également faire comme si il n'était pas incongru dans la situation. 


\section{Le minotaure, le corps-machine et la danseuse}

24 Trois exemples sur le terrain de Corps dessinant, complèteront cette analyse des conditions de l'observation filmée du travail des relations sociales : trois séquences de Performers\#10 qui rendent compte de trois situations de travail différentes, produisant chacune d'elle un projet de performance artistique.

La première situation concerne la préparation d'une performance qui mêle la nudité des corps à un bestiaire baroque, associant notamment une tête de faisan et un masque de taureau-cyclope: Metamorpher - Nid de Phonix et Minotaure. Les deux artistesplasticiens, Hélène Barrier et Julien Salaud, souhaitent tourner une séquence vidéo introductive à leur performance. Pour cela, ils se retrouvent en forêt de Sologne, face à un immense chêne, majestueux et multi-centenaire, ouvert comme un éventail et pouvant les accueillir sur ses puissantes branches. Leur projet consiste à évoluer à moitié nu sur l'arbre, véritable architecture végétale, tels des êtres hybrides, mihumain mi-animal, rappelant la figure du chaman. Ils se filmeront chacun leur tour avec une tablette numérique. Nous les accompagnons sur leur lieu de travail. En cette belle matinée de fin novembre, il fait aussi froid que le ciel est bleu... Hélène Barrier et Julien Salaud se déshabillent. Elle se couvre juste d'une peau de bête; quant à lui, il revêt une longue robe de femme. Dans le lointain, ils entendent un faisan alors qu'ils se réchauffent avec le thé brûlant qu'Hélène Barrier a apporté dans un thermos.

La circonstance d'engagement est très fine en raison de son caractère peu conventionnel. Toute situation humaine est socialement cadrée, de telle sorte que les acteurs sociaux sont institués dans des cadres de légitimation selon des modes de justification éprouvés, en tant que leurs actions sont reconnues par le sens commun (nous retrouvons, ici, les notions de convenable et de convenientia précédemment évoquées). Par exemple, l'intervention médicale d'urgence s'appuie sur une légitimité socialement très puissante en tant que l'engagement dans cette action vaut pour le bien d'autrui. En revanche, le caractère subversif de l'acte performatif d'Hélène Barrier et de Julien Salaud relève du monde inspiré de la création artistique (Boltanski et Thévenot, 1997 : 200-206) qui se justifie par le désir de création, l'originalité et la singularité de son rapport au monde. Il convenait donc de prendre en considération les modes de justification par lesquelles ces deux personnes pouvaient légitimer leur action. En l'espèce, leur cadre de légitimation sociale était soutenu par le projet du Musée des Arts et Métiers dans lequel s'inscrivait leur création artistique. Or, notons que notre présence contribuait précisément à mettre en représentation le cadre légitime de leur action puisque l'anthropologie filmée que nous réalisions était produite par cette institution muséale. Il convient, également, de préciser que l'arbre sur lequel les artistes venaient évoluer se situait dans une propriété privée tenue par des amis de Julien Salaud, qui avaient donné leur accord pour les besoins de cette performance. Ce détour par les modes de justification de l'action permet de souligner les conditions autorisant l'observation filmée d'une situation sociale. La compréhension de ce cadre de légitimation est essentielle pour s'insérer dans le cadre d'engagement des personnes (filmées) dans leur action.

27 Pour autant, cette approche compréhensive ne suffit pas bien sûr au maintien $d u$ rapport filmant-filmé. En l'espèce, bien que nous étions parfaitement intégrés à la circonstance d'engagement (Hélène Barrier et Julien Salaud nous avaient embarqués dans leur voiture depuis Paris pour rejoindre la Sologne), nous devions faire preuve 
d'attention pour se maintenir dans la délicate épaisseur de cette singulière situation de travail: une maladresse, un moment d'inattention, une remarque déplacée, une malencontreuse contrainte de tournage, etc. et le fragile équilibre de la situation risquait de se rompre. Voici le premier échange de la séquence d'ouverture du premier épisode de Performers\#10. Julien Salaud et Hélène Barrier s'apprêtent à rejoindre le grand chêne pour réaliser leur séquence vidéo :

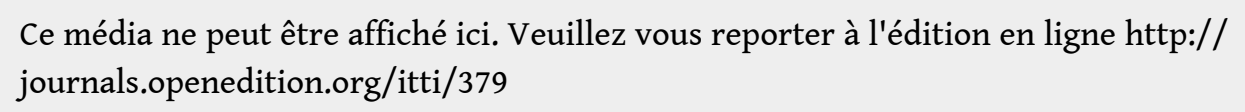

Épisode 1:02'01“-03’05”

Assis sur le sol, Julien Salaud et Hélène Barrier trinquent avec leur tasse de thé

J. Salaud - Nostrovia !17

H. Barrier - Nostrovia!

Pendant qu'ils prennent leur thé, un faisan s'entend au loin...

J. Salaud - Ah, c'est un faisan!

H. Barrier - Où est-ce qu'il y a un faisan?

J. Salaud - Là bas...

Hélène Barrier essaie de voir l'oiseau pendant que Julien allume une cigarette.

La cigarette aux lèvres, Julien Salaud s'apprête à monter sur l'arbre et interpelle Hélène Barrier.

J. Salaud - Bon, on est prêt ! ? Tu montes aussi ?..

Hélène Barrier, recroquevillée sur l'arbre, acquiesce très doucement par des murmures.

J. Salaud - Monsieur le chêne, je monte!

Julien Salaud monte sur une branche, à côté d'Hélène Barrier enveloppée dans sa peau de bête.

J. Salaud - Bon je vais faire le guignol sur la branche!

H. Barrier - Tout à fait (sur un ton tranquille).

Julien Salaud s'avance sur la branche pour faire le cochon-pendu: le corps à l'envers, la robe glisse et recouvre le haut du torse et la tête, dénudant le bas du corps et les jambes croisées sur la branche d'arbre. Pendant ce temps Hélène Barrier se déplace lentement sur le chêne, juste couverte d'une peau de bête sur le dos et de son masque de minotaure sur la tête.

La description de cette scène et la retranscription de l'échange conversationnel entre Hélène Barrier et Julien Salaud montrent en quoi cette situation n'allait pas de soi : dès lors, c'est bien le travail de la fabrication de la performance qui devenait l'objet de l'observation filmée et non l'étrangeté, voire la bizarrerie, de la situation. Il s'agissait donc de rendre compte non pas tant de la performance elle-même (alors encore en travail) que de la performativité des interactions sociales entre les deux artistes engagés dans la fabrication de leur œuvre artistique. Pour cela, il s'agissait de porter son attention sur le cadre de légitimation de la circonstance d'engagement, autrement dit sur la mise en représentation de l'engagement des acteurs dans la conduite de leur action.

31 En raison de la nudité des corps, la singularité de cette situation de travail supposait que l'attention de l'observateur-filmant se porte bien sur la valeur d'engagement des acteurs dans la circonstance de travail et non sur l'intérêt que pouvait représenter la situation comme objet filmique. Sinon, la situation de tournage aurait pu être dénoncée par les personnes filmées comme un acte de voyeurisme ou, plus simplement, se limitant à une forme d'instrumentalisation de la scène représentée pour les besoins du film: cette situation de tournage, sur la fabrication d'une performance, ne fait que 
radicaliser la disposition ordinaire de l'observateur-filmant s'attachant à rendre compte d'une situation de travail. Il convient toujours de savoir apprécier le délicat jeu de cadres d'engagement qui est à l'œuvre dans l'observation filmée d'une activité humaine, entre le cadre social de la situation de travail et le cadre théâtral de ce qui est représenté par la production du travail lui-même. Une telle préoccupation peut apparaître excessive, voire spéculative, tant une situation ordinaire se donne à voir comme l'état naturel des choses. Pourtant, il est indispensable de savoir s'ajuster à l'objet d'attention des personnes filmées, tout en restant attentif à ses propres exigences techniques de tournage. Filmer une situation de travail implique de se tenir en tension entre ces deux objets d'attention - ce qui est filmé et l'acte de filmer - et sans les confondre afin d'éviter bien des déconvenues sur un terrain.

En l'espèce, le simple fait de s'attacher à la situation de travail des performers permet de se concentrer sur l'engagement des acteurs dans la fabrication de la situation et non dans la simple captation d'une situation qui serait donnée à voir. Seule l'intentionnalité de l'observateur-filmant peut soutenir cette distinction des cadres et ainsi éviter des malentendus dans le rapport filmant-filmé. Pour cela, il convient de porter son attention sur l'objet de la circonstance d'engagement : autrement dit, ce pour quoi les personnes agissent et interagissent dans une situation sociale donnée.

Le deuxième exemple, extrait de ce terrain de Performers\#10, concerne une toute autre situation de travail, structurée par le cadre d'action précisément défini de la performance: dans l'ex-sacristie de l'ancienne église du Musée des Arts et Métiers, l'artiste-plasticienne Romina de Novellis prépare sa performance avec le groupe de participants qui l'accompagne. Pendant trois heures, ils devront rester debout, sur une estrade dans une des salles du musée, quasiment immobiles : leurs actions se limitant à reproduire inlassablement le même geste mécanique des mains, évoquant le tissage d'un fil. Cette performance sur la mécanique des corps, intitulée $A D N$, se déroulera en miroir de vieilles machines du XIX ${ }^{e}$ siècle exposées dans la même salle. Avant de se rendre sur le lieu de la performance, Romina de Novellis prodigue ses conseils et rappelle quelques consignes aux artistes participants qui devront tenir trois heures durant :

Ce média ne peut être affiché ici. Veuillez vous reporter à l'édition en ligne http:// journals.openedition.org/itti/379

\section{Épisode 5 : $21^{\prime} 03^{\prime \prime}-211^{\prime} 53$ ”}

Tenez, tenez, tenez ! Jusqu'à... che la Madona vi accompagna ${ }^{18}$ (rires) !... Vous avez tous une expérience corporelle, donc vous comprenez ce que je suis en train de dire. Plus ou moins, vous avez tous une expérience corporelle. Vous avez entendu dire çà. Donc, vous savez ce que c'est. Il faut tenir. Plus vous tenez, plus la fin elle est facile. Plus on se laisse aller... et plus çà devient une catastrophe, cette histoire (rires) !...

Donc, jambes ouvertes... De toutes façons, je vais vous montrer, hein! Vous ne buvez plus. Vous avez bien mangé ? Des protéines?

Filmer la performance, c'est filmer le corps. La dimension performative d'une situation de travail correspond au cadre corporel de ce qui se joue entre les acteurs : percevoir la performativité d'une situation humaine revient à s'attacher à sa corporéité, autrement dit en tant qu'elle procède du corps humain. Pour cela, on s'attachera tout d'abord à la parole qui fait du corps humain un corps symbolisant (Vasse, 1977 : 91-92): en effet, ce 
qui est dit (le discours) ne peut s'exprimer qu'en tant qu'il est symbolisé par ce corps qui parle. La parole est l'expression même du caractère irrévocable de l'engagement chez un humain, comme en témoigne de nombreuses expressions et proverbes telles que "engager sa parole", "tenir parole", "Qui parle beaucoup, doit dire des sottises $»^{19}$. Mais, cette corporéité résulte bien sûr également des gestes et postures (que nous avons évoqués dans le cas de la situation de travail de l'agent EDF), des attitudes et des représentations du corps (selon qu'il soit plus ou moins habillé ou dénudé), ainsi que des formes de sociabilité résultant des attachements et de proximité entre les personnes (Hall, 1978). Ces quatre vecteurs de la corporéité d'une situation de travail structurent précisément la performance de Romina de Novellis, en tant qu'ils sont mis à l'épreuve par l'irrévocabilité du temps de l'action: pendant trois heures, dans le silence absolu (donc, « sans parole ») les performers répètent les mêmes gestes selon une immuable posture et une mise en scène de la représentation des corps au travail. Cette mécanique corporelle joue doublement en miroir avec son environnement (proxémie): à la fois par la co-présence immobile des machines exposées et par les déplacements du public qui doit s'adapter à cette présence bordant l'allée du Musée. Filmer cette performance supposait de percevoir ces différents éléments composant la circonstance d'engagement de la situation de travail des performers. Sinon, cela revenait tout simplement - là, aussi - à faire la captation d'une représentation artistique.

Troisième et dernier exemple, issu de Performers\#10, illustrant la pratique de l'observation filmée d'une situation de travail: l'échange introductif à la dernière répétition de la performance Corps, Graph, Erg de Cécile Proust et de Jacques Hœpffner, en présence de Nathalie Giuliana-Peyrard, alors en charge de ce projet Corps dessinant au sein du Musée des Arts et Métiers ${ }^{20}$.

Ce média ne peut être affiché ici. Veuillez vous reporter à l'édition en ligne http:// journals.openedition.org/itti/379

\section{Épisode 5 : 02'20“ - 03'12"}

Nathalie Giuliana entre dans une salle du Musée où Cécile Proust s'apprête à répéter sa performance avec son collaborateur, Jacques Hœpffner. Elle marche d'un pas énergique et assuré.

N. Giuliana - Bonjour Cécile! J'arrive avec la Tour Eiffel! Ça va? (Elle s'approche pour

l'embrasser).

C. Proust - Non, je suis malade...

N. Giuliana - Ah bon!

C. Proust - Ah bah, ouais... désolé. Ça va?

N. Giuliana - Très bien!

C. Proust - Très bien?

N. Giuliana - Ouais.

C. Proust - Bon cool... Bon euh, tu peux te mettre en bas... mais tu peux te mettre en haut aussi... (C. Proust désigne la mezzanine) puisque tu es quand même, euh...

Nathalie Giuliana s'en va d'un pas énergique et assuré. Elle revient par le balcon de la mezzanine et arrive à la hauteur de Jacques Hœpffner. Tout en marchant, elle s'adresse aux deux performers.

N. Giuliana - Bon, Cécile et Jacques...

J. Hœpffner - (off) Oui.

N. Giuliana-Quand les gens arriveront et que Cécile performera, n'hésitez pas à les inviter à monter.

Nathalie Giuliana arrive à la hauteur de Jacques Hœpffner puis se pose au-dessus de 
Cécile Proust, en s'appuyant sur la balustrade en verre de la Mezzanine.

C. Proust - (off) Oui, oui... C'est prévu!

N. Giuliana - Parce que spontanément, ils n'oseront pas forcément...

C. Proust - (off) Oui, oui... Mais, c'est prévu! (regardant en hauteur, en direction de N. Giuliana) Je vais les accueillir de toutes façons et j'vais leur dire!

N. Giuliana - (off) Super!

C. Proust - C'est bon, on attend personne...!? (Elle se tourne vers la caméra). C'est bon pourvous?

(En plongée depuis la mezzanine) Cécile Proust commence la chorégraphie de sa performance, sous le regard attentif de Nathalie Giuliana, accoudé à la balustrade et de Jacques Hœpffner qui prend des photos. une de ces circonstances banales, en mode mineur (Piette, 1992), souvent enchâssées entre deux temps forts - deux actions majeures - et que l'on remarque à peine habituellement. Bien souvent, ce type d'échanges entre parenthèses, où se joue les salutations et les ajustements réciproques avant ou après une circonstance d'engagement, est perçue comme un temps mort. Pour une équipe de tournage, c'est habituellement l'occasion de faire une pause et/ou de s'occuper du matériel, du dispositif technique : une telle interaction, très convenue, ne suscite donc pas d'emblée l'attention et ne motive pas à rester concentré pour tenir le cadre de l'observation filmée.

41 Pourtant, l'ethnographie de cet échange montre combien une telle micro action donne à comprendre ce qui se joue entre les acteurs, en termes de rapports de territoire, de reconnaissance des places de chacun, d'affirmation des légitimités et des cadres institutionnels, entre l'artiste-plasticienne, la représentante du musée et le collaborateur-photographe. Percevoir toute cette épaisseur sociale, chargée de la production symbolique des échanges ${ }^{21}$, suppose de se déprendre de l'état naturel des choses : autrement dit, de suspendre un rapport au monde où ce qui se passe semble aller de soi. Il convient de solliciter son étonnement pour faire advenir « l'intuition de l'instant » (Bachelard, 1992). Selon cette approche phénoménologique, il devient alors possible d'observer la dimension performative d'une micro-circonstance d'engagement, tel que ce moment de salutation et d'ajustements où se travaillent les places de chacun : s'y manifeste la production symbolique des échanges, ce qui se joue entre les acteurs. Cette situation qui nous semblait si familière nous devient alors très singulière et suscite notre attention, notre désir de filmer.

Mais comment filmer cette circonstance introductive à la répétition d'une performance, alors même qu'elle survient en dehors du cadre prescrit de la situation de travail proprement dite? Pour cela, il s'agit de porter l'attention, précisément, au travail des relations sociales qui sont à l'œuvre dans la production des situations instituées; autrement dit, à la fabrication des situations qui sont convenues dans un cadre institutionnel donné. Une telle disposition conduit alors à anticiper les microsinteractions susceptibles de donner à comprendre ce qui s'y joue.

En ce qui nous concerne, lors du tournage de Performers\#10, cela c'est traduit en termes techniques par la décision de placer un micro HF sur la personne susceptible de se déplacer entre les acteurs en raison de son autorité institutionnelle: c'était le cas le Nathalie Giuliana-Peyrard. En conséquence, la preneuse de son et moi-même, à la caméra, étions en mesure de filmer l'arrivée à l'improviste de la représentante du 
musée et de pouvoir rendre compte de l'instant de salutation avec la performeuse Cécile Proust.

Mais, une autre difficulté allait surgir : comment se placer pour filmer cette interaction alors que la situation se déroulait dans un espace relativement complexe puisqu'il se divisait entre la salle proprement dite et une mezzanine où se situait déjà le collaborateur de Cécile Proust, Jacques Hœpffner. J’avais déjà repéré cette contrainte quand Nathalie Giuliana-Peyrard a décidé de monter sur la mezzanine, sous la proposition de Cécile Proust, afin de regarder la performance. Reste qu'il me fallait décider immédiatement où j'allais me positionner pour filmer la suite de l'interaction dans cette configuration singulière du lieu de l'échange : est-ce que je devais suivre la représentante du musée ou rester auprès de la performeuse ou encore adopter une position tierce? En fait, il convenait de ne pas s'éloigner de Cécile Proust pour les besoins de la prise de son qui s'effectuait, sur elle, avec une perche. Le temps que Nathalie Giuliana monte les escaliers la conduisant à la mezzanine, je me suis ajusté avec la caméra pour cadrer son arrivée, dès qu'elle parviendrait sur le balcon. Restait ensuite à filmer l'échange qui s'annonçait entre Nathalie Giuliana en haut de la mezzanine et Cécile Proust en bas dans la salle. Là aussi, il s'agissait de s'adapter à la circonstance d'engagement et aux contraintes techniques : le choix fut donc de rester en contre-plongée sur Nathalie Giuliana afin d'anticiper sa prise de parole, en recueillant en off les réponses de Cécile Proust. Ensuite, j'ai cadré Cécile Proust - avec les réponses de Nathalie Giuliana en off - dans la mesure où l'ordre de l'interaction me laissait supposer que la performeuse clôturerait l'échange avant le début de sa propre répétition. Cette possibilité d'anticiper les échanges suppose de s'accorder au rythme de la situation sociale observée, sinon la caméra risque de balayer frénétiquement la situation sans pouvoir stabiliser son cadre et de ne pouvoir rendre compte de l'ordre de l'interaction.

Voilà comment cette petite scène d'une minute a mobilisé à la fois une approche ethnographique des interactions sociales et une pratique de l'observation filmée: ce modeste échange - produit dans l'interstice d'une dense journée de workshop - donne à percevoir ce qui se joue entre l'autorité de l'institution muséale représentée par Nathalie Giuliana et la légitimité de l'action de la performeuse qui instruit son territoire dans les locaux mêmes du Musée. Tout se déroule dans une parfaite cordialité, malgré les rapports de pouvoir qui se jouent entre les interactants. En effet, leur relation ne cesse de faire valoir la place de chacun, selon des jeux de rôles où se négocient des revendications de statut: tout commence avec l'arrivée de Nathalie Giuliana apportant la maquette de la Tour Eiffel. Par ce geste attentionné, elle fait de la performeuse son obligée dans une relation de familiarité et sollicite sa reconnaissance à l'égard du musée. Mais, Cécile Proust lui annonce qu'elle est malade et qu'en vertu du principe de précaution pour ne pas lui transmettre le virus, elle lui refuse l'embrassade de bienvenue. Puis, paradoxalement, alors qu'elle est elle-même souffrante, la performeuse vient à demander par deux fois, à Nathalie Giuliana, si elle va bien... renversant ainsi la relation de sollicitude. S'ensuit un échange où la performeuse induit (instruit) la bonne place à la représentante du musée pour qu'elle puisse regarder sa performance avant la représentation publique : s'accordant à la proposition, Nathalie Giuliana rejoint la (sa) place d'un pas martial. A peine arrivée à la hauteur du collaborateur de Cécile Proust, sur la mezzanine, elle rappelle quelques consignes quant à la présence des personnes venant assister à la représentation. En convoquant ainsi la figure du public, la représentante du musée rappelle à la performeuse qu'elle est liée à 
l'institution, au-delà des relations interpersonnelles, par un tiers-terme qui fait autorité. De fait, elle restaure ainsi l'échange dans le cadre conventionnel qui réunit musée et performers.

La performeuse clôture ce micro-échange avec la représentante du Musée par une interrogation conclusive qui laisse entendre que l'on n'attend plus personne et que l'on peut donc commencer la performance. Cette clôture est renforcée par un regard caméra et un terme d'adresse à l'observateur-filmant lui demandant : "c'est bon pour vous ». Notons que tout regard-caméra résulte d'une sortie du cadre d'engagement de la personne filmée (Lallier, 2009 : 157-164) : en l'espèce, cette interaction permet à la performeuse de faire-valoir que le cadre d'engagement relatif à l'échange qui vient de se dérouler est bien terminé et qu'elle contrôle la conduite de sa situation de travail.

\section{Conclusion}

L'observation filmée des interactions sociales ne suffit pas à rendre compte d'une situation de travail par le film documentaire d'observation: autrement dit, sans commentaires off ni interview. Faut-il encore que les situations sociales observées puissent faire récit par le suivi des échanges entre les acteurs. Sinon, l'observation filmée consiste simplement en une technique d'enregistrement favorisant la microanalyse des interactions et dont la compréhension résulte du décryptage produit, par exemple, par des chercheurs en socio-linguistique, en anthropologie ou en ethnométhodologie.

La compréhension d'une séquence d'observation filmée procède soit de commentaires exogènes à l'action représentée (voix-off, interviews, entretiens) soit des commentaires endogènes, c'est-à-dire des propos tenus entre les acteurs de la situation. Quelque soit le type de commentaires, la forme narrative résultera de la mise en évidence des enjeux qui sous-tendent la situation représentée, autrement dit ce qui est en jeu pour les acteurs, ce qu'ils ont à perdre ou à gagner par leur investissement dans l'action. Dans le cas d'une pratique narrative de l'observation filmée, il conviendra donc de pouvoir rendre compte de ce qui se joue entre les acteurs en s'attachant à ce qu'ils (se) disent... Et ceci, de telle sorte que leurs échanges puissent donner à comprendre les enjeux qui motivent leurs engagements dans la situation sociale observée. On s'intéressera par exemple, aux modes de justification par lesquelles les acteurs légitiment leurs actions, comme nous l'avons souligné avec la situation de travail des deux artistes, Hélène Barrier et Julien Salaud. Plus largement, on sera attentif à ce qui est vécu en tant que circonstance singulière, historiquement située.

49 La forme narrative procède de ce qui ne va pas de soi, de ce qui est en travail, de ce qui apparaît comme une ressource ou une menace à la réussite de l'action. Par exemple, pour le dire plus simplement, le sprint final d'une course à pied produit un effet narratif immédiat là où la marche urbaine des passants dans la rue pourrait sembler de peu d'intérêt. La narrativité de l'action résulte de l'irréversibilité du temps. Au début de Temps et Récit, Paul Ricœur induit cette corrélation entre la forme narrative et la temporalité de l'action : «Le monde déployé par toute œuvre narrative est toujours un monde temporel [...]. Le temps devient humain dans la mesure où il est articulé de manière narrative; en retour le récit est significatif dans la mesure où il dessine les traits de l'expérience temporelle.» (Ricœur, 1991: 17). La perception de ce moment éphémère et singulier que constitue «l'expérience temporelle » renvoie précisément à 
cet état d'irréversibilité du temps de l'action et dessine, par là-même, la forme narrative de sa représentation.

C'est en cela qu'il devient possible de rendre compte d'une situation humaine par l'observation filmée du travail des interactions sociales: en tant qu'elle est une expérience temporelle. Précisons que cette pratique de l'observation filmée, visant à rendre compte d'une situation par ce que (se) disent les acteurs entre eux, doit s'interpréter comme un principe méthodologique de l'enquête de terrain : elle ne doit pas pour autant constituer un dogme intangible interdisant l'emploi des autres formes $\mathrm{du}$ film documentaire. Dans Nioro-du-Sahel, une ville sous tension, par exemple, j'ai utilisé parfois la forme de l'interview afin de mettre en perspective un arrière-plan permettant d'enrichir la compréhension d'une situation observée. Pour Performers\#10, j'ai inséré des cartons dans les séquences d'observation filmée, afin de préciser quelques éléments de contexte. En effet, pour que le spectateur soit attentif à ce qui se joue dans une séquence d'observation filmée, il convient qu'il ne soit pas obligé d'essayer de comprendre ce qui se passe.

\section{BIBLIOGRAPHIE}

Austin, John L., Quand dire, c'est faire, Paris, Seuil, « Points Essais », 1970.

Bachelard, Gaston, L'intuition de l'instant, Paris, Stock, Le livre de poche, 1992.

Boltanski, Luc, thévenot, Laurent, De la justification. Les économies de la grandeur, Paris, Gallimard, NRF Essais, 1997.

Certeau, Michel (de), L'absent de l'histoire, Paris, Mame, coll. Repères, 1973.

Dejours, Christophe, Le facteur humain, Paris, PUF, « Que sais-je ?», 1995.

Foucault, Michel, Les mots et les choses, Paris, Gallimard, « Tel », 1990.

Goffman, Erving, La mise en scène de la vie quotidienne, tome1, La présentation de soi, Paris, Minuit, «Le sens commun », 1996.

Goffman, Erving, Les cadres de l'expérience, Paris, Minuit, « Le sens commun », 1991, p. 98.

Hall, Edward T., La dimension cachée, Paris, Seuil, Points-Essais, 1978.

Jankélévitch, Vladimir, L'irréversible et la nostalgie, Paris, Flammarion, « Nouvelle Bibliothèque Scientifique », 1974

Lallier, Christian, « Le cirque, la performance du contre-rôle de soi », in Les lieux du cirque, Christine Fourmaux (dir.), Paris, Éditions Le Manuscrit, 2008.

Lallier, Christian, Pour une anthropologie filmée des interactions sociales, préface de Jean-Paul Colleyn, Paris, Éditions des Archives Contemporaines, 2009.

Passeron, René, La naissance d'Icare. Éléments de poḯtique générale, Valenciennes, Ae2cq Éditions, Presses Universitaires de Valenciennes, 1996. 
Piette, Albert, Le mode mineur de la réalité : Paradoxes et photographies en anthropologie, Louvain-LaNeuve, Peeters, BCILL, 1992.

Ricœur Paul, Temps et récit, Tome 1, Paris, Seuil, « Points Essais », 1991.

Alfred Schütz, Le chercheur et le quotidien, Phénoménologie des sciences sociales, trad. Anne NoschisGilliéron, Paris, Méridiens Klincksieck, 1994.

Vasse Denis, Le temps du désir, essai sur le corps et la parole, Paris, Seuil, coll. Points Essai, 1997.

Vatin François, « De la naissance de la psychologie appliquée au débat sur le taylorisme, autopsie d'un échec : le cas français (1890-1920) », in Les histoires de la psychologie sociale, sous la direction d'Yves Clot, Toulouse, Octarès Éditions, 1996.

\section{NOTES}

1. Lorsque je pratique l'observation filmée, j'utilise toujours la prise casque de la caméra afin de filmer en écoutant la situation à partir des interactions enregistrées par la prise de son.

2. Cf. Dictionnaire Le Petit Robert: Contrôle. n. m. (1422 ; contre-rôle "registre tenu en double", Paris, 1986, p. 385).

3. La contredanse, par exemple, s'effectue par quatre danseurs qui se font face en exécutant les mêmes figures, mais dans un mouvement opposé. Le contrepoint correspond en musique à un motif secondaire qui se superpose au thème majeur de la composition, en ayant une réalité propre. Jouer en contrepoint consiste à suivre une mélodie mais en l'interprétant simultanément et indépendamment, comme une sorte d'accompagnement.

4. Cette notion d'intimité fait référence au caractère privatif d'une situation de travail. En effet, lorsqu'une situation de travail s'organise elle privatise le lieu même où elle va s'exercer : quand des agents EDF, par exemple, interviennent sur la voie publique ils disposent des barrières de sécurité entourant l'espace de l'intervention avec ce panneau «Chantier interdit au public». De même, dans un restaurant, la porte conduisant aux cuisines peut signaler l'interdit d'accès avec l'inscription « Privé ».

5. Il convient de préciser que l'activité de tournage constitue, en soi - comme toute situation de travail -, une circonstance d'engagement. Tout l'enjeu de l'observation filmée, consiste donc à ce que cet engagement (du filmant) puisse s'enchâsser dans la circonstance d'engagement de la personne (filmée) dans son action, de telle sorte que c'est en tant qu'elle agît que cette dernière peut intégrer la présence du filmant sans pour autant modifier son action.

6. La notion même de travail désigne à la fois le produit réalisé et la réalisation du produit. Cette double interprétation fut mise en évidence par la physiologie qui distingua l'effort produit et le produit de l'effort : ce dispositif de catégorisation a permis d'étudier le rapport entre "énergie dépensée » et « résultat obtenu » - au sens d'un beau ou d'un mauvais travail (Vatin, 1996: 50). Notons que cette distinction entre effort produit et produit de l'effort, fut à l'origine de l'invention de l'enregistrement par images animées comme en atteste le Chronophotographe mis au point précisément par un physiologue travaillant sur la représentation de l'effort du corps en mouvement : Jules-Etienne Marey (1830-1904).

7. Dans Les mots et les choses, Michel Foucault rappelle que les notions de «convenable " et de "convenance» désignent une forme de lien entre les personnes selon un principe de ressemblance avec le voisinage : « la "convenientia" est une ressemblance liée à l'espace dans la forme du "proche en proche". Elle est de l'ordre de la conjoncture et de l'ajustement. [...] Sont "convenantes" les choses qui, approchant l'une de l'autre, viennent à se jouxter; elles se touchent du bord, leurs franges se mêlent, l'extrémité de l'une désigne le début de l'autre. [...] Par ce voisinage, l'âme reçoit les mouvements du corps, et s'assimile à lui, tandis que "le corps 
s'altère et se corrompt par les passions de l'âme". Dans la vaste syntaxe du monde, les êtres différents s'ajustent les uns aux autres. [...] La ressemblance impose des voisinages qui assurent à leur tour des ressemblances » (Foucault, $1990: 33$ ).

8. Une personne "se laisse" filmer dans l'observation de son action dès lors que sa mise en représentation vaut pour la valeur de son investissement dans la situation sociale représentée. Cette condition de possibilité à l'observation filmée d'une personne dans son action suppose que l'observateur-filmant fasse comme si il n'était pas incongru à se tenir dans une situation à laquelle il n'a pas à participer comme acteur ratifié par les échanges : autrement dit, au sens où, précise Isaac Joseph, "le participant ratifié est celui qui est à sa place dans l'ordre de l'interaction 》 (Joseph, $1998: 66)$.

9. Alfred Schütz (1899-1959) est un philosophe considéré comme le fondateur d'une sociologie phénoménologique.

10. Cf. L'ethnographie visuelle de Christian Lallier consacrée au Kalash du Pakistan, sur le site internet: c-lallier-anthropologie-filmee.com. On pourra également se référer à l'ouvrage de l'anthropologue Jean-Yves Loude, Kalash. Les derniers "infidèles" de l'Hindu-Kush, avec la collaboration de Viviane Lièvre, photographies d'Hervé Nègre, Paris, édition Berger-Levrault, coll. Espace des hommes, 1980.

11. Ce projet artistique fut coproduit par le Musée des Arts et Métiers (CNAM), dirigé à l'époque par Yves Winkin, et l'Institut ACTE : un laboratoire de recherche sur l'art, de l'Université Paris-1 Panthéon Sorbonne et du CNRS, dirigé également à l'époque par Richard Conte, artiste-plasticien et Professeur d'Université. Les performances ont été réalisées avec l'équipe de recherche EsPAS dirigée par Barbara Formis (Maître de conférences à l'Université Paris-1). EsPAS constituait une des équipes de recherche de l'UMR ACTE, regroupant plusieurs artistes-chercheurs. Précisons que l'Institut ACTE n'est plus une UMR aujourd'hui.

12. Performers\#10 - La fabrique de l'éphémère est une anthropologie filmée réalisée selon la pratique de l'observation des interactions sociales avec Mélodie Drissia Tabita en tant que preneuse de son et assistante de réalisation. Cette série documentaire de $3 \mathrm{~h} 30$ en sept épisodes s'attache à rendre compte de la fabrication de dix performances artistiques présentées au Musée des Art \& Métiers à Paris, lors d'un week-end intitulé Corps dessinant. Pour cela, Performers\#10 fait le récit du travail de ce projet artistique, au cours du work-shop préparatoire et durant le week-end des performances. Les sept épisodes nous entraîne dans la fabrique de ces représentations qui n'existeront que le temps d'un week-end. Sans voix-off, le film vise à nous immerger dans les relations sociales de travail entre les acteurs qu'ils soient artistes, techniciens ou représentants des institutions concernées (musée et laboratoires de recherche de l'Université Paris 1). Performers\#10 se termine par le débriefing qui clôture cette expérience et où se révèle la complexité d'une telle entreprise. Cette anthropologie filmée a été produite par le Lab'-AF : le Laboratoire d'anthropologie filmée dirigé par Christian Lallier et la vidéaste Mélodie D. Tabita.

13. En ce qui concerne ma pratique d'anthropologie filmée, l'expression " observateur-filmant » désigne une équipe de deux personnes, incluant l'anthropologue-réalisateur caméraman et une preneuse de son assistante de réalisation. La prise de son suppose une mixette et trois micros dont un micro directionnel et deux micros HF. Depuis L'élève de l'Opéra, la vidéaste Mélodie D. Tabita assure la prise de son sur mes documentaires.

14. Le cadre de cet article ne permet pas de développer, ici, les conditions de cette disposition. Il conviendrait, notamment, de mentionner les incidences de forme que peut parfois produire la présence de l'observateur-filmant: soit, une certaine formalisation dans la tenue des échanges qui se traduit par une parole et des attitudes un peu plus soutenues, en raison de la réflexivité de la personne filmée vis-à-vis de l'observateur-filmant (Lallier, 2009 : 117). Ces incidences de forme peuvent s'estomper, voire disparaître, en raison de l'intensité de l'engagement des personnes dans leur action et/ou par l'intégration de l'observateur-filmant dans le cadre social de la situation observée. Par ailleurs, notons que ces incidences de forme s'apparentent à l'effet 
cathartique que peut produire la présence de la caméra ou, plus précisément, par l'intensité de l'attention exprimée par l'observateur-filmant sur la situation sociale observée.

15. Rappelons que l'on ne peut s'engager dans deux circonstances à la fois : ainsi lorsque nous souhaitons répondre à un appel téléphonique alors que nous sommes déjà engagés dans un échange, il convient de s'excuser ou du moins de prendre en considération la personne avec qui nous avions un échange afin de suspendre momentanément cette circonstance d'engagement et de pouvoir nous engager dans la conversation téléphonique. En revanche, ces deux engagements conversationnels peuvent ne former qu'une seule circonstance si l'échange téléphonique participe de la discussion des deux personnes en présence. De même, un nouveau cadre d'engagement (Goffman, 1991) pourra inclure implicitement la personne avec qui nous avions un échange dans notre conversation téléphonique, au principe qu'elle comprendra cette interruption momentanée de la situation préalablement en cours. Selon une analyse goffmanienne, nous pourrions même supposer que cette manière d'inclure implicitement notre interlocuteur dans l'échange téléphonique vise à interagir comme si nous avions une relation de familiarité avec lui alors que nous venons tout juste de le rencontrer (cf. notions de modulation et de fabrication (Goffman, 1991).

16. C'est précisément pour cette raison que nous n'emploierons pas l'expression « filmeur-filmé » qui induit une relation technique à un opérateur. Le gérondif utilisé dans le mot composé «filmant-filmé » désigne un lien d'interdépendance entre celui qui filme et celui qui est filmé, tous deux unis par une même situation sociale, objet de leur engagement partagé.

17. «Santé ! ( (en russe)

18. "Autant que la Vierge pourra vous accompagner». Retranscription et traduction de l'italien par l'auteur.

19. Selon la remarque d'Alfred Schütz, citée précédemment, la parole en tant qu'acte de langage est irrévocable, au sens où elle relève d'un travail (d'expression) qui modifie le monde.

20. A l'époque du projet, Nathalie Giuliana-Peyrard était responsable de la Cellule des projets artistiques et narratifs et de l'observatoire des publics et des tendances du Musée des Arts et Métiers-CNAM à Paris.

21. La production symbolique des échanges recouvre les cadres de légitimation, les formes de reconnaissances, les modes de justification de l'action, l'économie de la réputation... tout ce qui participe à la valeur des individus dans leur relation en face-à-face et qui constitue l'enjeu de l'échange, autrement dit ce qui est à perdre ou à gagner, tel que "perdre la face » ou «faire bonne figure ».

\section{RÉSUMÉS}

Filmer une situation de travail, c'est filmer ce qui se travaille dans une situation. Si on s'intéresse au travail du potier, on s'attachera à la glaise qui se travaille sur le tour. Dans une perspective interactionniste, il s'agira de s'attacher aux relations sociales produisant la situation humaine observée : autrement dit, aux formes d'engagement par lesquelles les personnes se représentent dans le cadre d'une interaction selon la conduite de leur rôle. En portant son attention sur ce travail des relations sociales, il devient alors possible de filmer ce qui se joue dans une situation de travail et d'en rendre compte sous une forme narrative par l'observation filmée des échanges. 
To film a work situation is to film what is taking place in a situation. If we focus on the potter's work, we will turn our attention to the clay getting worked on the pottery wheel. In an interactionist perspective, we will take a closer look at the social relations creating the observed human situation : in other words, to the forms of commitment through which people represent themselves in the setting of an interaction in accordance with the conduct of their role. By focusing on this work of social relations, it is then possible to film what is happening in a work situation and to account for it in narrative form through the filmed observation of the exchanges.

\section{INDEX}

Mots-clés : interactions, anthropologie filmée, circonstance d'engagement, travail des relations sociales, rapport filmant-filmés

Keywords : interactions, filmed anthropology, circumstances of commitment, work of social relations, relation between people filming and people being filmed

\section{AUTEUR}

\section{CHRISTIAN LALLIER}

Christian Lallier est anthropologue et cinéaste. Il a créé le Laboratoire d'anthropologie filmée, le Lab'AF : une structure indépendante qu'il dirige avec la vidéaste-ethnographe Mélodie Drissia Tabita. Le Lab'AF vient de réaliser une anthropologie filmée sur le patrimoine immatériel de la plus ancienne raffinerie de France, près de Rouen. Après une thèse soutenue en 2004 à l'EHESS il devient membre de l'Institut interdisciplinaire d'anthropologie du contemporain (iiAC - CNRS/ EHESS). Il a réalisé de nombreux films documentaires en anthropologie filmée, notamment avec Arte et le CNRS Images : Changement à Gare du Nord; Nioro-du-Sahel, une ville sous tension; La quatrième dimension de l'architecte; L'élève de l'Opéra, Performers\#10... Il a publié Pour une anthropologie filmée des interactions sociales aux Editions des Archives Contemporaines (2009). Ses travaux de recherche, l'ont conduit à enseigner à l'ENS de Lyon, à l'EHESS et à Sciences-Po Paris. Il enseigne depuis cinq ans à l'Université Grenoble-Alpes. 